
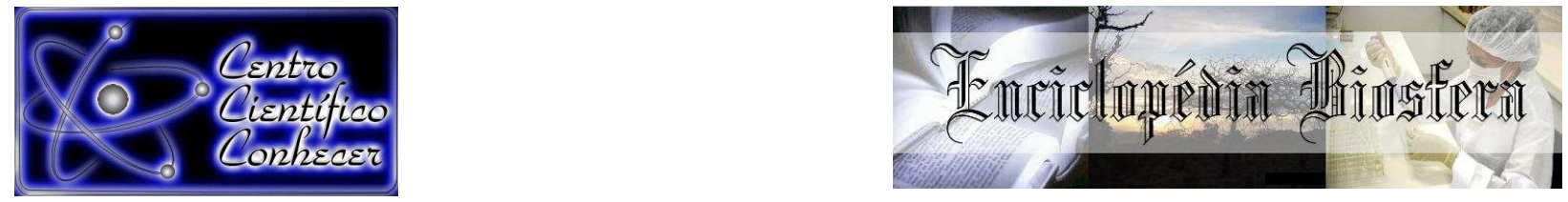

\title{
ESTOCAGEM EM CARBONO EM PORTAS INTERNAS DE MADEIRA DE EDIFÍCIOS RESIDENCIAIS
}

José de Almendra Freitas Junior ${ }^{1}$, Carlos Roberto Sanquetta ${ }^{2}$; Mateus Niroh Inoue Sanquetta $^{3}$; Ana Paula Dalla Corte ${ }^{2}$; Felipe Taroh Inoue Sanquetta ${ }^{4}$

1. Doutorando em Engenharia Florestal, Professor da Universidade Federal do Paraná Curitiba, Brasil (freitasjose@terra.com.br)

2. Eng. Florestal, Dr., Professor da Universidade Federal do Paraná

3. Acadêmico de Engenharia Florestal da Universidade Federal do Paraná

4. Acadêmico de Arquitetura e Urbanismo da Universidade Positivo

Centro BIOFIX de Pesquisas em Biomassa e Sequestro de Carbono

Universidade Federal do Paraná - UFPR

\section{Recebido em: 08/09/2015 - Aprovado em: 14/11/2015 - Publicado em: 01/12/2015} DOI: http://dx.doi.org/10.18677/Enciclopedia_Biosfera_2015_152

\begin{abstract}
RESUMO
Este artigo avalia o impacto de uma Porta Interna de Madeira (PIM) como material fixador de carbono e, portanto redutor das emissões de $\mathrm{CO}_{2}$ pelas obras de construção. $\mathrm{O}$ estudo desenvolveu medições de carbono em dois tipos usuais de Portas Internas de Madeira PIM completas (folha, batente e alizares), que são produzidas com madeira serrada e processada. Os batentes e alizares foram estudados a partir de amostras lineares. Já para as folhas, que são montadas com diferentes composições ao longo da altura e da largura, sem apresentar uma seção contínua, foi desenvolvida uma metodologia que se obteve amostras representativas da folha inteira de $700 \times 2100 \mathrm{~mm}$ analisando somente $900 \mathrm{~cm}^{2}$. Cabe salientar que a fixação de uma unidade de massa de carbono representa a não emissão de 3,66 unidades de massa de $\mathrm{CO}_{2}$, tendo em vista que a massa do átomo de carbono representa apenas $27,3 \%$ da massa molecular. As diversas espécies de madeira contêm de 40 a $45 \%$ de sua massa em carbono. Este estudo mostra que uma PIM completa, com massa de $37 \mathrm{~kg}$ contém aproximadamente $15 \mathrm{~kg}$ de carbono e evita a emissão de $54,9 \mathrm{~kg}$ de $\mathrm{CO}_{2}$ para a atmosfera. Inventários corporativos de emissões de publicados por construtoras mostram uma emissão de $\mathrm{CO}_{2}$ da ordem de $166 \mathrm{~kg}$ por $\mathrm{m}^{2}$ de construção (EVEN, 2013). A conclusão é que se considerarmos um apartamento residencial de três dormitórios de $60 \mathrm{~m}^{2}$, que possua um conjunto de oito PIM, é que estas fixam aproximadamente $4,41 \%$ do correspondente às emissões totais de $\mathrm{CO}_{2}$ da sua construção.
\end{abstract}

PALAVRAS-CHAVE: Estocagem de carbono, produtos de madeira, portas, construção.

\section{CARBON STORAGE IN INTERNAL WOOD DOORS OF RESIDENTIAL BUILDINGS}

\section{ABSTRACT}

This article assesses the impact of Internal Wood Doors such as carbon fixation material and therefore reducing $\mathrm{CO}_{2}$ emissions by the construction works. The study was 
developed for carbon measurements, with two usual types of complete sets of internal wooden doors (leaf, frame and doorjambs), which are produced in sawn wood or processed wood. The frames and doorjambs were studied from linear samples. As for the leafs, which are mounted with different compositions over the height and width without providing a continuous section, a methodology which was obtained to extract a representative samples of whole leaf $700 \times 2100 \mathrm{~mm}$ analyzing only $900 \mathrm{~cm}^{2}$ was developed. It should be noted that the setting of a unit mass of carbon is equal a not issuance of 3.66 mass units of $\mathrm{CO}_{2}$, in order that the carbon atom of the mass is only $27.3 \%$ of the molecular weight. The various wood species contain 40 to $45 \%$ of its mass on carbon. This study shows that a complete Internal Wood Door with mass of approximately $37 \mathrm{~kg}$, contains $15 \mathrm{~kg}$ of carbon and avoids the emission of $54,9 \mathrm{~kg}$ of $\mathrm{CO}_{2}$ to the atmosphere. Corporate inventories of construction emissions, shows a $\mathrm{CO}_{2}$ emissions of around $166 \mathrm{~kg}$ per $\mathrm{m}^{2}$ of construction (EVEN, 2013). The conclusion is that if we consider a residential apartment three-bedroom $60 \mathrm{~m}^{2}$, that has a set of eight Internal Wood Doors, is that these sets approximately correspond to $4.41 \%$ of total $\mathrm{CO}_{2}$ emissions of its construction.

KEYWORDS: Carbon storage, wood products, doors, construction.

\section{INTRODUÇÃO}

A produção de edifícios, o processo de fabricação e o transporte de materiais de construção impactam muito o ambiente (YAN et al., 2010). Segundo dados do Instituto Worldwatch, a construção de edifícios consome anualmente $40 \%$ da pedra, areia e cascalho, $25 \%$ de madeira e $16 \%$ de água em todo o mundo (ARENA \& DE ROSA, 2003).

A construção civil também é notoriamente conhecida como grande geradora de $\mathrm{CO}_{2}$, além de outros gases causadores do efeito estufa (GEE). As emissões desses GEE pela construção civil estão associadas à extração das matérias-primas usadas nos materiais de construção, aos processos de fabricação desses materiais, à energia dispendida durante o ciclo de vida da obra, à operação e manutenção da obra, além da disposição final dos resíduos e do transporte de materiais (BESSA, 2010). Este é um setor que consome muita energia, além de usar materiais altamente emissores de carbono durante a sua produção, como o cimento, o aço e a cal.

Os inventários de GEE em obras de edifícios no Brasil podem seguir as especificações do GHG Protocol - Corporate Accounting and Reporting Standard (GHG PROTOCOL, 2015; SANQUETTA et al., 2013a). Essa metodologia somente considera os materiais de construção de madeira e derivados, tais como portas e pisos, como fontes de emissões, contabilizando-se as emissões decorrentes de sua produção e transporte.

Ainda não se considera no Brasil, nestes tipos de obras, o potencial destes materiais como fonte de fixação ou estocagem de carbono, o que permitiria um aperfeiçoamento da metodologia. Ao invés de serem emissores, esses materiais de madeira e derivados poderiam ser tratados como elementos redutores das emissões de $\mathrm{CO}_{2}$ de uma obra. A madeira pode ser um substituto renovável e com funções análogas a outros materiais mais intensos em emissão de GEE usados na construção civil. Além de ser excelente como material de construção, a madeira é um fixador conhecido de carbono, elemento químico que compõe aproximadamente $45 \%$ da sua massa (OLIVEIRA, et al., 2011). Devido à composição da molécula de dióxido de carbono com dois átomos de oxigênio e um único átomo de carbono, (carbono tem massa molar igual a 12 e oxigênio massa molar 16), para cada unidade de massa de carbono fixada de forma permanente em uma edificação evita que 3,66 unidades de massa de $\mathrm{CO}_{2}$ sejam liberadas para a atmosfera. Portanto, o potencial da madeira produzida sustentavelmente, quando utilizada em materiais de construção que permanecerão na edificação por muitos 
anos, é muito grande. Alguns autores colocam a vida útil de uma porta entre 50 a 150 anos (MENET \& GRUESCU, 2012).

A armazenagem de carbono, que seqüestrado em produtos de madeira durante a vida útil, dependa da massa do produto e do o teor de carbono na biomassa, que varia dentro e entre espécies. Em geral considera-se a o teor de carbono em $50 \%$ do peso seco da madeira como um valor médio razoável (SATHRE, 2007). Estudo da Universidade do Tennessee considera para uma porta de madeira maciça (externa) genérica, a estocagem de 27,1 kg de carbono ou não emissão de 100,4 kg de $\mathrm{CO}_{2}$ (BERGMAN, et al., 2011). Este estudo examina alguns tipos de portas internas de madeira (PIM), que são mais leves e utilizadas em maior quantidade em edifícios que as externas, com vistas a contabilizar o carbono fixado nas suas esquadrias e usar esse valor no contexto dos levantamentos de emissões de dióxido de carbono $\left(\mathrm{CO}_{2}\right)$ da construção de edifícios residenciais no Brasil.

As tecnologias usuais de portas internas são virtualmente iguais no Brasil, nos Estados Unidos e na Europa (NBR 15.930-1 e 2/2011; WDMA I.S.1A-04/2004 e DIN 18101:2014-08). Portanto, os valores de fixação de carbono obtidos são válidos para todas estas regiões. Assim, o objetivo deste trabalho é quantificar a estocagem de carbono em portas internas de madeira, com vistas a apontar para maior uso dessa matéria-prima e aumentar a sustentabilidade ambiental de edificações residenciais.

\section{MATERIAL E MÉTODOS}

\section{Componentes de uma Porta Interna de Madeira (PIM)}

A PIM completa é composta de: marco, folha, alizares (interno e externo) é mostrada na Figura 1 (NBR 15.930-1, 2011).

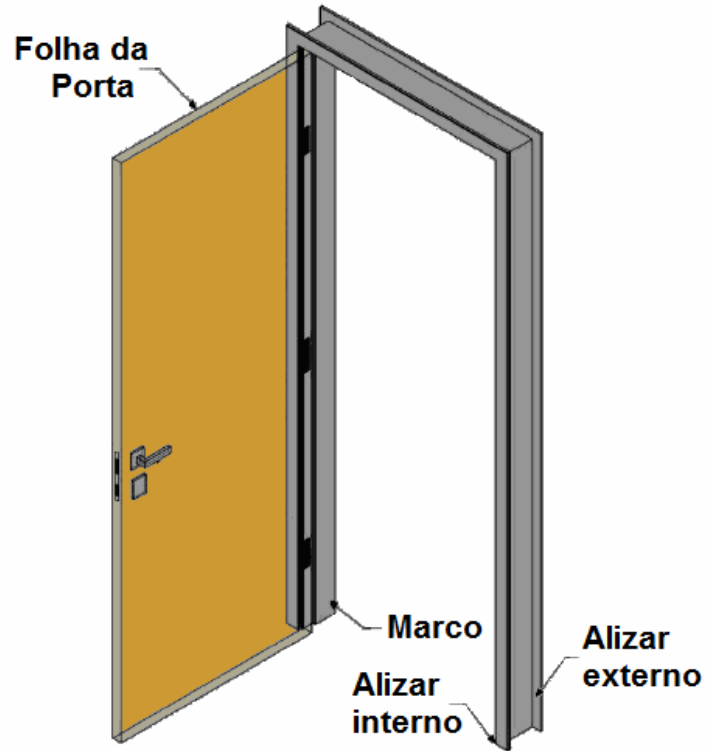

FIGURA 1. Os componentes de uma porta interna de madeira (PIM)

O marco é a estrutura do conjunto, ou seja, a parte da porta que é fixada nas paredes de vedação vertical (alvenarias, drywall, etc.) e sobre o qual a folha da porta é presa por meio de dobradiças (porta pivotante). O marco é composto por dois montantes verticais e uma travessa horizontal. A parte do marco que contém a movimentação da porta chama-se batente, onde pode ser colocada tira de amortecedor de borracha ou 
outro material resiliente para amortecer o choque e melhorar a vedação (NBR 15.930-1,2, 2011). Os marcos de madeira são usualmente produzidos em diversas espécies de madeira, tais como eucalipto ou pinus, compensados, MDF (Medium-Density Fiberboard) ou EGP (Edge Glued Panel).

A folha de porta é o painel plano móvel, constituído de um quadro de madeira com reforços, um núcleo (vazado ou sólido) e duas capas coladas a um quadro e preenchida por um núcleo formando um conjunto rígido. O quadro é a estrutura periférica da folha da porta, composta de montantes verticais e horizontais em madeira geralmente pinus ou eucalipto. Os reforços são peças de madeira inseridas no quadro ou no núcleo da folha da porta para a fixação de ferragens e/ou acessórios. O núcleo é o enchimento da folha ou o material presente no interior da folha da porta, com a função de estruturar as capas e, eventualmente, adequar seu desempenho como isolante acústico a NBR 15.575-4/2013. O núcleo pode ser vazado ou maciço. Os vazados são constituídos de um conjunto de sarrafos de madeira ou alveolado de papelão, os maciços em aglomerado, EGP ou eventualmente poliestireno expandido. Um núcleo vazado sarrafeado é composto por tiras ou sarrafos que são dispostos horizontalmente ou verticalmente de forma aleatória. Os sarrafos proporcionam a estabilidade das capas. Um núcleo em aglomerado ou EGP torna, em geral, a folha mais pesada proporcionando um melhor isolamento acústico. As capas da folha da porta são as chapas planas que fazem o fechamento das superfícies principais da folha. Nas PIM podem ser chapas de compensados, chapas duras (Hard Board) ou chapas de MDF (Medium-Density Fiberboard).

Os alizares, um conjunto de três partes de cada lado da esquadria, são as peças empregadas para cobrir as juntas existentes entre a parede e o marco, são também conhecidas por guarnição, vistas, molduras ou cobre-juntas (NBR 15.930-1, 2011). Os alizares para pintura de madeira e derivados, são usualmente produzidos em madeira maciça, MDF (Medium-Density Fiberboard) ou EGP (Edge Glued Panel). (NBR 15.930-1 e 2/2011; WDMA I.S.1A-04/2004 e DIN 18101:2014-08).

\section{PIM Analisadas}

Para este levantamento foram escolhidas, entre diversos fornecedores do mercado, duas folhas de portas de modelos diferentes, as quais atendem os padrões médio e leve da NBR 15.930-1/2011. Estas folhas possuem dois núcleos diferentes, um com preenchimento de aglomerado e outro com preenchimento em sarrafeado. Estes dois núcleos atendem a NBR 15.575-4/2013 para diferentes padrões de isolamento acústico. Para todos os conjuntos, foi selecionada a dimensão da folha de $700 \times 2100 \mathrm{~mm}$ por ser a mais usual e que representa uma média das demais dimensões utilizadas, com a espessura de $35 \mathrm{~mm}$, seguindo os padrões de PIM adequadas para tráfego moderado segundo a NBR 15.930-1,2/2011. Estas dimensões também representam as médias deste tipo de esquadrias em mercados da Europa e dos EUA. Ambas as folhas de portas são para acabamento em pintura.

Completando os componentes das esquadrias, foram escolhidos dois tipos de conjuntos diferentes de marcos com alizares, também usuais pela indústria da construção civil. Os conjuntos de marcos e alizares estudados são um em EGP de pinus, sendo o marco com capas de chapa de MDF e o outro com marco em eucalipto maciço e alizar em MDF, todos para acabamento em pintura. As Figuras 2 e 3 mostram os dois tipos diferentes de folhas de portas estudados nesta pesquisa. É interessante observar que as espécies utilizadas nos produtos estudados são originárias de reflorestamentos, portanto, madeira sustentável. 

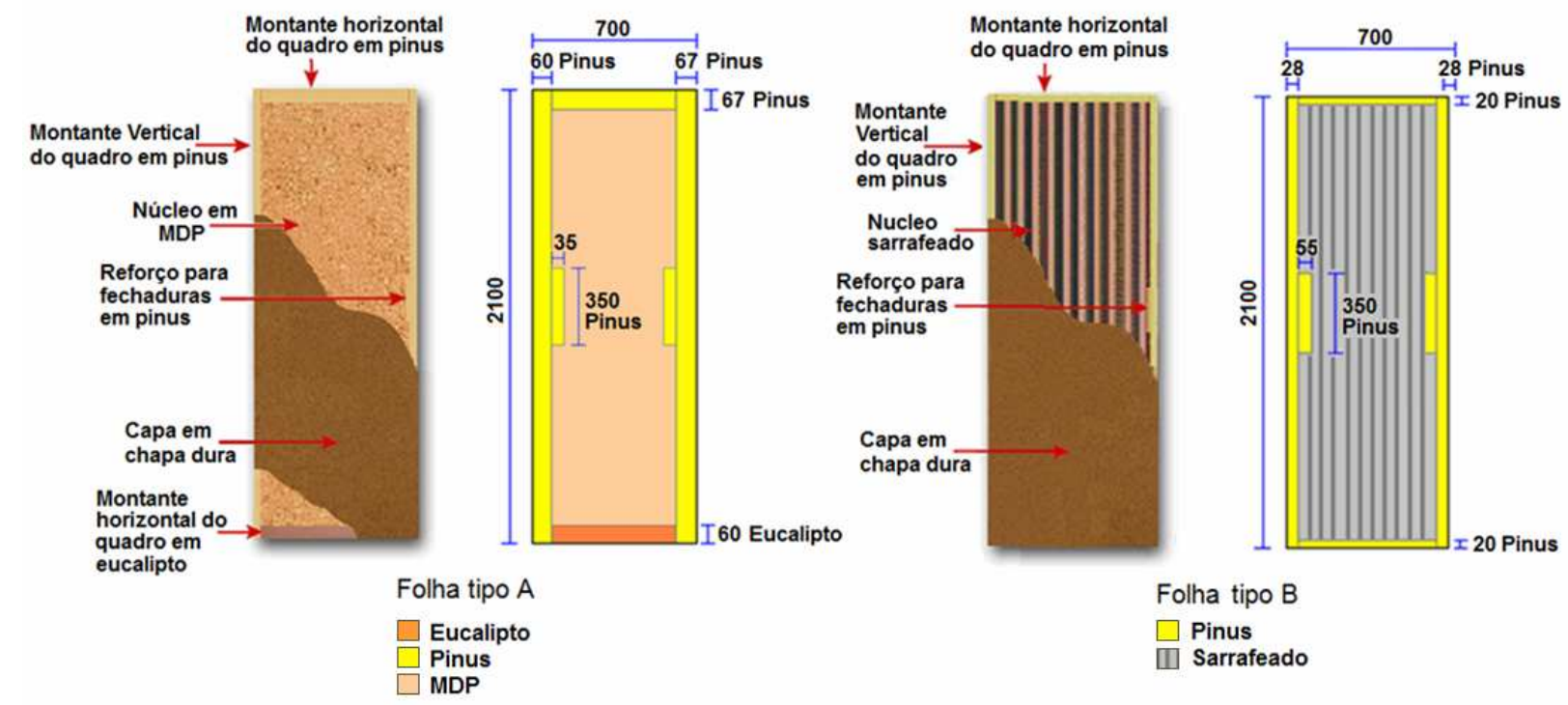

FIGURA 2. Folha de porta tipo $A$ com quadro em pinus e eucalipto, núcleo em aglomerado e capas em chapa dura e B com quadro em pinus, núcleo sarrafeado e capas em chapa dura. Dimensões em mm.

MARCO (A)

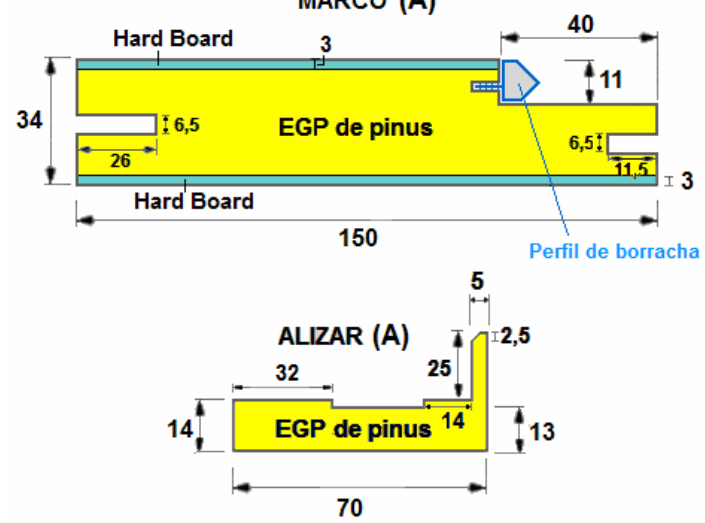

MARCO (B)
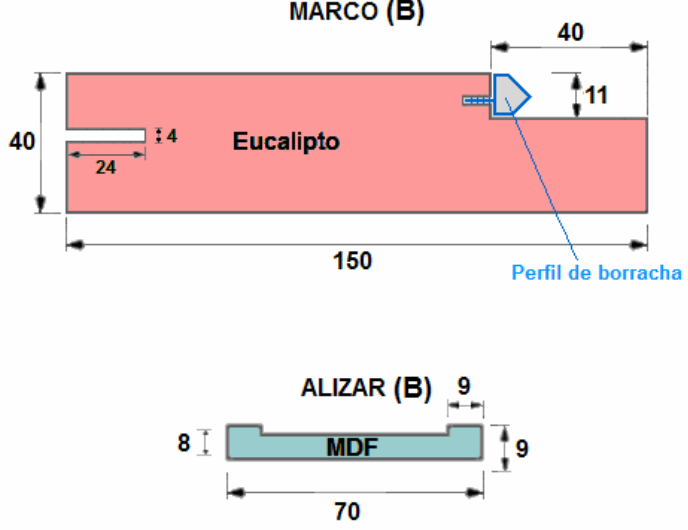

FIGURA 3. Seções dos dois marcos diferentes estudados, tipo A com marco e alizares em EGP de pinus com capas de chapa de MDF e tipo B com marco em eucalipto e o alizar em MDF. As dimensões estão marcadas em mm

\section{Preparação das Amostras e Determinação dos Teores de Carbono}

As PIM são compostas pelas folhas das portas, os marcos e os alizares, como mencionado previamente, para este estudo foram criadas metodologias de amostragem com a finalidade de calcular a massa de carbono existente nas partes de madeira e derivados que permitisse a avaliação das massas de carbono presentes nos diferentes componentes das esquadrias.

Inicialmente, foram determinadas as massas médias dos diferentes componentes das PIM selecionadas (Tabela 1). Para estas medições foram utilizadas quatro unidades de folhas de portas, duas de cada tipo ( $A$ e $B$ ), dois conjuntos de marcos de cada tipo (um A e um B), assim como dois conjuntos de alizares (um A e um B). Observa-se que para um mesmo vão livre interno da esquadria, os comprimentos dos marcos e dos alizares são influenciados pelas suas espessuras. 
TABELA 1. Massas médias em umidade de equilíbrio, medidas para as folhas das portas, os marcos e alizares completos para uma esquadria

\begin{tabular}{ccc}
\hline Material & Dimensões $(\mathrm{mm})$ & Massa média $(\mathrm{g})$ \\
\hline Folha A & $35 \times 700 \times 2.100$ & 21.116 \\
Folha B & $35 \times 700 \times 2.100$ & 16.810 \\
Marco A & Seção $\times 4.968$ & 12.837 \\
Marco B & Seção x 4.980 & 17.679 \\
Alizar A & Seção x 10.216 & 5.517 \\
Alizar B & Seção x 10.240 & 4.506 \\
\hline
\end{tabular}

Para os marcos a metodologia separou como frações representativas para amostragem, dois trechos lineares de cada marco (A e B) com $1.000 \mathrm{~mm}$ cada. Destes foram extraídas duas partes de $250 \mathrm{~mm}$ como amostra final para ser triturada. Para os alizares, que tem seções bem menores, considerou-se como frações representativas para amostragem trechos lineares de $500 \mathrm{~mm}$. Estas amostras foram pesadas em umidade de equilíbrio e secas. Depois foram trituradas em três equipamentos diferentes até alcançar a dimensão de pó, com as partículas passando na peneira mesh 10. Esta redução foi à necessária para que posteriormente fosse possível determinar os teores de carbono.

Para as folhas das portas a metodologia de amostragem criada foi diferente devido ao fato das folhas das portas serem uma montagem com diferentes materiais, e seus interiores conterem muitos vazios, ao contrário dos marcos e alizares, que são praticamente maciços.

As folhas são compostas por materiais diferentes no quadro de reforço perimetral, no enchimento, nos reforços internos e nas capas. Observando-se a montagem das amostras das folhas das portas, verificou-se que só seria possível desmontá-las completamente desagregando-as na água, técnica que dificultaria a determinação das massas de cada material devido à variação do teor de umidade.

A limitação da capacidade do equipamento de trituração não possibilitou moer portas inteiras. A metodologia criada para a amostragem das folhas, foi a de se dividir a área das portas em amostras de $900 \mathrm{~cm}^{2}$ para cada folha, de tal forma que cada amostra contivesse as diferentes partes das folhas nas mesmas porcentagens que uma folha inteira.

As amostras foram pesadas em umidade de equilíbrio e secas, sendo posteriormente trituradas e homogeneizadas em pó, com as partículas passando na peneira mesh 10, para que fosse possível determinar os teores de carbono.

As densidades básicas das amostras foram medidas conforme a NBR 11.941/2003. As análises de teores de carbono foram realizadas em amostras de $0,350 \mathrm{~g}$, que foram pesadas em uma barquinha de combustão de material cerâmico.

Na determinação de carbono a amostra é submetida à combustão empregando-se ambiente de oxigênio puro a $1350^{\circ} \mathrm{C}$. A amostra passa por um processo de redução oxidativa que faz com que os compostos que contenham carbono quebrem e o liberem. A partir do sistema de combustão, os gases passam através de um sistema, para remover a umidade. Uma célula de detecção de infravermelho mede a concentração de dióxido de carbono. O instrumento converte estes valores em porcentagem, usando uma equação predefinida no software que leva em conta o peso da amostra, a calibração e o valor de umidade conhecida. O analisador empregado foi o modelo LECO C-144 (LECO CORPORATION, 2008), instrumento projetado para medir o teor de carbono em materiais orgânicos e inorgânicos. Foram feitas 4 repetições de cada amostra para obter-se as 
respectivas estatísticas (média, desvio padrão e coeficiente de variação em percentagem).

\section{RESULTADOS E DISCUSSÃO}

\section{Massas e Densidades}

$\mathrm{Na}$ Tabela 2 estão apresentados os resultados das massas e densidades das amostras das folhas das portas, dos marcos e alizares. Observa-se que há diferenças dimensionais entre os marcos e alizares, tanto no tocante aos volumes quanto às massas, o que repercute em diferenças de densidade básica. Esses valores de densidade, por sua vez, repercutem em maior ou menor estoque de carbono, haja vista que quanto maior a densidade mais carbono estará estocado por unidade de volume da porta. Nota-se também a menor densidade básica das folhas das portas em comparação com as correspondentes dos marcos e alizares.

TABELA 2. Massas e densidades das amostras das folhas, marcos e alizares das PIM

\begin{tabular}{ccrrrrc}
\hline Material & Amostra & $\begin{array}{c}\text { Volume } \\
\left(\mathrm{cm}^{3}\right)\end{array}$ & $\begin{array}{c}\text { Massa em } \\
\text { umidade de } \\
\text { equilíbrio }(\mathrm{g})\end{array}$ & $\begin{array}{c}\text { Massa } \\
\text { seca } \\
(\mathrm{g})\end{array}$ & $\begin{array}{c}\text { Perda } \\
\text { média de } \\
\text { massa }(\%)\end{array}$ & $\begin{array}{c}\text { Densidade } \\
\text { básica }\left(\mathrm{g} / \mathrm{cm}^{3}\right)\end{array}$ \\
\hline Folha A1 & 1 & 3.150 & $1.278,8$ & $1.138,3$ & 12,3 & 0,361 \\
& 2 & 3.150 & $1.298,2$ & $1.158,8$ & 12,0 & 0,368 \\
Folha A2 & 1 & 3.150 & $1.349,3$ & $1.196,1$ & 12,8 & 0,380 \\
& 2 & 3.150 & $1.245,0$ & $1.105,6$ & 12,6 & 0,351 \\
Folha B1 & 1 & 3.150 & $1.069,0$ & 960,5 & 11,3 & 0,305 \\
& 2 & 3.150 & $1.036,8$ & 932,0 & 11,2 & 0,296 \\
Folha B2 & 1 & 3.150 & 991,0 & 896,3 & 10,6 & 0,285 \\
Marco A & 1 & $1.104,06$ & $1.020,0$ & 919,5 & 10,9 & 0,292 \\
& 2 & $1.104,06$ & 620,7 & 563,5 & 12,8 & 0,510 \\
Marco B & 1 & $1.366,00$ & 906,8 & 778,3 & 13,1 & 0,497 \\
& 2 & $1.366,00$ & 832,3 & 719,9 & 15,6 & 0,570 \\
Alizar A & 1 & 541,44 & 254,7 & 223,8 & 13,8 & 0,527 \\
& 2 & 541,44 & 284,3 & 252,3 & 12,7 & 0,413 \\
Alizar B & 1 & 289,00 & 217,9 & 196,7 & 10,8 & 0,681 \\
& 2 & 289,00 & 217,3 & 196,8 & 10,4 & 0,681 \\
\hline
\end{tabular}

\section{Teores de Carbono}

O teor de carbono é a porcentagem da massa de carbono presente na biomassa total da madeira. Os teores de carbono dos componentes das PIM variaram de 41 a $44 \%$ (Tabela 3). As variações foram da ordem de 2 a $6 \%$ em termos de CV\%. O teor de carbono foi menor na folha da PIM de aglomerado em comparação com a de sarrafeado. Isso possivelmente se deve à maior participação de madeira maciça no segundo caso. Outro fator que pode influenciar é a espécie, pois diferentes espécies apresentam distintos teores de carbono na madeira e nos outros órgãos da planta (DALLAGNOL et al., 2011; SANQUETTA et al., 2013b). 
TABELA 3. Médias, desvio padrão e coeficiente de variação dos teores de carbono das amostras das folhas das portas.

\begin{tabular}{crrrrrr}
\hline Estatística & \multicolumn{1}{c}{ Folha A } & Folha B & Marco A & Marco B & Alizar A & Alizar B \\
\hline Média & 41,508 & 44,733 & 44,190 & 44,601 & 44,242 & 44,242 \\
Desvio padrão & 2,513 & 0,868 & 1,728 & 1,083 & 1,436 & 1,573 \\
CV\% & 6,05 & 1,96 & 3,86 & 2,43 & 3,24 & 3,24 \\
\hline
\end{tabular}

\section{Estoques de carbono}

Obtidos os teores de carbono unitários dos materiais, para se calcular as massas de carbono dos componentes das PIM, foram computadas as médias das porcentagens de carbono em relação à densidade do material em umidade de equilíbrio (seco ao ar) e seco em estufa. A partir dos teores percentuais de carbono em relação às massas em umidade de equilíbrio e as massas totais dos componentes das esquadrias foram calculadas as massas por componentes e as respectivas massas em carbono (Tabela 5).

TABELA 4. Densidades médias das folhas, marcos e alizares e teores de carbono dos componentes das PIM

\begin{tabular}{cccccc}
\hline Material & $\begin{array}{c}\text { Densidade em } \\
\text { umidade de } \\
\text { equilíbrio } \\
\left(\mathbf{g . c m}^{-3} \mathbf{)}\right.\end{array}$ & $\begin{array}{c}\text { Densidade } \\
\text { seca em } \\
\text { estufa } \\
\mathbf{( g . c m}^{-3} \mathbf{)}\end{array}$ & $\begin{array}{c}\text { \%C da } \\
\text { massa em } \\
\text { umidade de } \\
\text { equilíbrio }\end{array}$ & $\begin{array}{c}\text { Massa total } \\
\mathbf{( g )}\end{array}$ & $\begin{array}{c}\text { Massa em } \\
\text { carbono } \\
\mathbf{( g )}\end{array}$ \\
\hline Folha A & 0,410 & 0,365 & 36,921 & 21.116 & 7.796 \\
Folha B & 0,327 & 0,294 & 40,300 & 16.810 & 6.774 \\
Marco A & 0,569 & 0,504 & 39,124 & 12.837 & 5.022 \\
Marco B & 0,637 & 0,549 & 38,433 & 17.679 & 6.794 \\
Alizar A & 0,498 & 0,440 & 39,076 & 5.517 & 2.155 \\
Alizar B & 0,753 & 0,681 & 38,474 & 4.506 & 1.733 \\
\hline
\end{tabular}

Para a esquadria tipo A (folha, marcos e alizares), totalizaram-se $14.974 \mathrm{~g}$ de carbono e para a esquadria tipo B, $15.302 \mathrm{~g}$. É importante observar, que embora os dois totais tenham chegado a valores semelhantes, as quantidades individuais dos componentes isolados são bem diferentes, e é perfeitamente possível utilizar a folha de porta $A$ com o conjunto marco $B$ e alizar $B$, o que poderia resultar em um total de $16.324 \mathrm{~g}$ de carbono, valor $17,0 \%$ superior ao da folha $\mathrm{B}$ em conjunto com o marco $\mathrm{A}$ e o alizar $\mathrm{A}$, que totaliza $13.951 \mathrm{~g}$ de carbono.

Outra informação relevante obtida com o estudo é que independentemente da mistura utilizada de materiais de madeira e seus derivados (MDF, MDP, Hard Board, etc.), o percentual de carbono contido é de aproximadamente $39 \%$ da massa do material. Portanto, para uma aproximação bastante razoável da quantidade de carbono retida por uma PIM, basta medir a massa da PIM e multiplicar por 0,39.

\section{Emissões de GEE evitadas}

Para se avaliar o impacto de uma PIM na redução do $\mathrm{CO}_{2}$ emitido por uma obra é necessário multiplicar a massa de carbono fixada em cada unidade por 3,66, como mencionado previamente. Esse novo valor dá o equivalente em $\mathrm{CO}_{2}$ que deixa de ser emitido pelo fato de o carbono estocado na porta ali permanecer por muito tempo. Portanto, uma PIM com massa de $15 \mathrm{~kg}$ de carbono evitaria a emissão de 54,9kg de $\mathrm{CO}_{2}$ equivalente fosse liberada para a atmosfera. 
Aprofundando a importância das PIM quanto redução das emissões das obras, considerando um apartamento residencial de três dormitórios comum de $60 \mathrm{~m}^{2}$, que possua um conjunto de oito portas, comparando com os resultados de geração de $\mathrm{CO}_{2}$ equivalente $165,93 \mathrm{~kg} \cdot \mathrm{m}^{2}$ por metro quadrado de construção (EVEN, 2013), deduz-se que somente os conjuntos das esquadrias de PIM podem fixar aproximadamente $4,41 \%$ do total correspondente às emissões totais de $\mathrm{CO}_{2}$ de uma obra de construção civil de edifícios de apartamentos residenciais.

Portanto, é verificável que a consideração de materiais de madeira e derivados, quando aplicados de forma permanente nas construções residenciais, aponta para significativos aperfeiçoamentos nos relatórios de emissões de $\mathrm{CO}_{2}$, alcançando reduções importantes nas emissões totais com a contabilização de aplicações usuais de materiais de madeira, como esquadrias, coberturas, pisos, entre outros. A substituição de materiais mais intensos em carbono, como cimento, aço, cal, tijolos cerâmicos alumínio, etc., por produtos de madeira repercute em evidente redução de emissões de GEE e obras civis.

\section{CONCLUSÕES}

- Os teores médios de carbono em portas internas de madeira usadas em construções residenciais estão na faixa de $39 \%$ da massa em umidade de equilíbrio e de $44 \%$ da massa seca em estufa;

- Uma PIM padrão pode conter cerca de $15 \mathrm{~kg}$ de carbono estocado em sua estrutura, evitando a emissão de 54,9kg de $\mathrm{CO}_{2}$ para a atmosfera;

- Conjuntos das esquadrias de PIM podem fixar aproximadamente $4,41 \%$ do total correspondente às emissões totais de $\mathrm{CO}_{2}$ de uma obra de construção civil de edifícios de apartamentos residenciais.

\section{REFERÊNCIAS}

ARENA, A.P.; C. DE ROSA. Life cycle assessment of energy and environmental implications of the implementation of conservation technologies in school buildings in Mendoza-Argentina. Building and Environment, v.38, n.2, p.359-368, 2003.

BESSA, V.M.T. Contribuição da metodologia de avaliação das emissões de dióxido de carbono no ciclo de vida das fachadas de edifícios e escritórios. 286 f. Tese (Doutorado em Engenharia de Construção Civil e Urbana), Escola Politécnica da Universidade de São Paulo, São Paulo, 2010.

BERGMAN, R.; PUETTMANN, M.; TAYLOR, A. Carbon Impacts of wood products. Center for Renewable Carbon, University of Tennessee Institute of Agriculture, 2011. Disponível em: <http://www.wwpinstitute.org/documents/CIWPpub.pdf> Acesso em 21/09/2015.

DALLAGNOL, F.S.; MOGNON.F; SANQUETTA, C.R.; DALLA CORTE, A.P. Teores de carbono de cinco espécies florestais e seus compartimentos. Floresta e Ambiente. v.18, n.4, p.410-416, 2011.

DIN 18.101:2014-08 - Türen - Türen für den Wohnungsbau - Türblattgrößen, Bandsitz und Schlosssitz - Gegenseitige Abhängigkeit der Maße, DIN - Deutsches Institut für Normung, 2014. 
EVEN. Relatório da Gestão de Carbono 2013. Disponível em: $<$ http://www.even.com.br/sustentavel/wp-content/uploads/2014/08/EVEN-RelatorioGestao-Carbono-2013-final.pdf >. Acesso em 31/07/2015.

GHG PROTOCOL. Método, $2015 . \quad$ Disponível em: http://www.ghgprotocolbrasil.com.br/metodo?locale=pt-br>. Acesso em: 31/07/2015.

LECO Corporation. C-144 Carbon Determinator - Specification Sheet. St. Joseph, Missouri, USA, 2008. Disponível em: $<$ http://www.usbiocolombia.com/files/organica/c144dr-hoja-tecnica.pdf> Acesso em 15/12/2014.

MENET, J., GRUESCU I. C..GOAL AND SCOPE DEFINITION INVENTORY ANALYSIS IMPACT ASSESSMENT COMPARATIVE LIFE CYCLE ASSESSMENT OF A BUILDING COMPONENT: CASE OF A FRONT DOOR. Proceedings 2nd LCA Conference, 6-7 November 2012, Lille $\quad$ France. $\quad$ Disponível em $<$ http://www.avnir.org/documentation/book/LCAconf menet 2012 en.pdf> Acesso em 28/09/2015.

NBR 11.941. Madeira - Determinação da densidade básica. ABNT Associação Brasileira de Normas Técnicas, 2003.

NBR 15.930-1. Portas de madeira para edificações. Parte 1 Terminologia e simbologia. ABNT Associação Brasileira de Normas Técnicas, 2011.

NBR 15.930-2. Portas de madeira para edificações. Parte 2. Requisitos. ABNT Associação Brasileira de Normas Técnicas, 2011.

NBR 15.575-4. Desempenho de edificações - Sistemas de vedações verticais internas e externas. ABNT Associação Brasileira de Normas Técnicas, 2013.

OLIVEIRA, E. O.; NAKAJIMA, N. Y.; CHANG M.; HALISKI, M.; Determinação da quantidade de madeira carbono e renda da plantação florestal. Documentos 220 EMBRAPA FLORESTAS, Colombo PR, 2011. Disponível em: $<$ http://www.infoteca.cnptia.embrapa.br/bitstream/doc/898993/1/Doc220.pdf > Acesso em 15/09/2015.

SANQUETTA, C.R.; FLIZIKOWSKI, L.C.; DALLA CORTE, A.P.; MOGNON, F.; MAAS, G.C.B. Estimativa das emissões de gases de efeito estufa em uma obra de construção civil com a metodologia GHG Protocol. Enciclopédia Biosfera, v.9, n.16, p.1088-1106, 2013a.

SANQUETTA, M.N.I.; SANQUETTA, C.R.; DALLA CORTE, A.P.; MOGNON, F.; MENDONÇA, V.C. Teores de carbono e densidade básica da madeira de Cryptomeria japonica (Thunb. ex L. F.) D. Don em povoamentos no município de Rio Negro - PR. Enciclopédia Biosfera, v.9, n.17, p.2450-2458, 2013b.

SATHRE, R. Life-Cycle Energy and Carbon Implications of Wood-Based Products and Construction. Ecotechnology and Environmental Science. Mid Sweden University Doctoral Thesis 34. Department of Engineering, Physics and Mathematics. Mid ENCICLOPÉDIA BIOSFERA, Centro Científico Conhecer - Goiânia, v.11 n.22; p.1192 2015 
Sweden University, Östersund, Sweden, 2007. Disponível em:<http://www.divaportal.org/smash/get/diva2:2032/FULLTEXT01.pdf> Acesso em 22/09/2015.

WDMA I.S.1A-04 - Industrial Standard for Architectural Wood Flush Doors. Window \& Door Manufacturers Association, 2004. Disponível em: http://www.maiman.com/Other/Maiman/Documents/Downloads/TF/WDMA\%201.S.\%201A. pdf> Acesso em: 21/09/2015.

YAN, H.Y.; QIPING, S.; FAN, L.C.H.; WANG, Y.; ZHANG, L. Greenhouse gas emissions in building construction: A case study of One Peking in Hong Kong. Building and Environment, v.45, n.4, p.949-955, 2010. 